\title{
ARTICLE
}

Molecular Diagnostics

\section{Individual inflammatory marker abnormalities or inflammatory marker scores to identify primary care patients with unexpected weight loss for cancer investigation?}

\author{
B. D. Nicholson (D) $^{1}$, J. L. Oke ${ }^{1}$, P. Aveyard ${ }^{1}$, W. T. Hamilton ${ }^{2}$ and F. D. R. Hobbs ${ }^{1}$
}

BACKGROUND: Combinations of inflammatory markers are used as prognostic scores in cancer patients with cachexia. We investigated whether they could also be used to prioritise patients attending primary care with unexpected weight loss for cancer investigation.

METHODS: We used English primary care electronic health records data linked to cancer registry data from 12,024 patients with coded unexpected weight loss. For each individual inflammatory marker and score we estimated the sensitivity, specificity, likelihood ratios, positive predictive value (PPV) and the area under the curve along with 95\% confidence intervals for a cancer diagnosis within six months.

RESULTS: The risk of cancer associated with two abnormal inflammatory markers combined in a score was higher than the risk associated with individual inflammatory marker abnormalities. However, the risk of cancer in weight loss associated with individual abnormalities, notably a raised C-reactive protein, was sufficient to trigger further investigation for cancer under current NICE guidelines.

CONCLUSIONS: If scores including pairs of inflammatory marker abnormalities were to be used, in preference to individual abnormalities, fewer people would be investigated to diagnose one cancer with fewer false positives, but fewer people with cancer would be diagnosed overall.

British Journal of Cancer (2021) 124:1540-1542; https://doi.org/10.1038/s41416-021-01282-4

\section{BACKGROUND}

The evidence base for using simple blood tests to identify symptomatic adults attending primary care at increased risk of cancer is almost entirely limited to tests used in isolation. ${ }^{1}$ With the exception of anaemia (for colorectal cancer) and jaundice (for pancreatic cancer) the risk of individual cancers associated with single blood test abnormalities is below the three percent threshold recommended by NICE for urgent cancer investigation. ${ }^{1}$ Thrombocytosis, hypercalcaemia and hypoalbuminaemia are associated with increased cancer risk across multiple sites. ${ }^{1}$ Combinations of abnormal tests may hold greater potential to rule-in and rule-out further cancer investigation but research is lacking on the most predictive combinations in symptomatic patients. ${ }^{2,3}$

Inflammatory marker scores are used by hospital specialists as surrogate markers of the catabolic drive in cancer cachexia. ${ }^{4}$ Raised scores correlate with the extent of weight loss (WL), predict symptom burden, tumour progression and survival across multiple cancer types. ${ }^{5}$ Commonly reported scores are the modified Glasgow prognostic score (mGPS), that combines low albumin and raised C-reactive protein (CRP), the neutrophil-lymphocyte score (NLS) and the platelet-lymphocyte score (PLS).

Unexpected WL may represent the first clinical manifestation of cancer in primary care. Cancer risk rises above 3\% when adults with unexpected WL also have low albumin or a raised white cell count, calcium, platelets or inflammatory markers. ${ }^{6}$ The aim of this study was to investigate whether inflammatory marker scores could be used to select patients with unexpected WL for further cancer investigation in preference to individual inflammatory marker abnormalities.

\section{METHOD}

We used primary care electronic health records from the Clinical Practice Research Datalink (CPRD), a representative anonymised English database, from between 1 January 2000 to 31 December 2012 and linked to the National Cancer Registrations and Analysis Service (NCRAS) cancer register. ${ }^{7}$ Patients were included if aged $\geq 18$ and registered for at least 12 months with a CPRD general practice before the index date. The index date was the first validated unexpected $\mathrm{WL}$ code. These codes equate to a mean weight loss of $5 \%$ or more within a 6 -month period. ${ }^{6}$ All patients had the following inflammatory markers recorded in the 3 months before to 1 month after the index date: albumin, CRP, lymphocyte count (LC), neutrophil count (NC) and platelet count (PC). We excluded patients with evidence of a past cancer diagnosis or a WL intervention within the previous six months. Cancers coded in the six months after the index date were identified in the CPRD and NCRAS data using an existing library of codes to include all

\footnotetext{
${ }^{1}$ Nuffield Department of Primary Care Health Sciences, University of Oxford, Oxford, UK and ${ }^{2}$ Medical School, University of Exeter, Exeter, UK
} Correspondence: B. D. Nicholson (brian.nicholson@phc.ox.ac.uk) 
high level ICD-O categories. ${ }^{8}$ Non-melanoma skin, in situ, benign, ill defined or uncertain cancers were not included.

The following thresholds were used for abnormal inflammatory markers: albumin $<35 \mathrm{~g} / \mathrm{l} ; \mathrm{CRP}>10 \mathrm{mg} / \mathrm{l} ; \mathrm{LC}<1.5 \times 10^{9} / \mathrm{l} ; \mathrm{NC}>7.5 \times$ $10^{9} / \mathrm{l} ; \mathrm{PC}>400 \times 10^{9} / \mathrm{l}$. The following inflammatory marker scores were derived: $\mathrm{mGPS}=2(\mathrm{CRP}>10 \mathrm{mg} / \mathrm{l}$ and albumin $<35 \mathrm{~g} / \mathrm{l})$; $\mathrm{NLS}=2\left(\mathrm{NC}>7.5 \times 10^{9} / \mathrm{l}\right.$ and $\left.\mathrm{LC}<1.5 \times 10^{9} / \mathrm{l}\right)$; and PLS $=2(\mathrm{PC}>$ $400 \times 10^{9} / \mathrm{I}$ and LC $\left.<1.5 \times 10^{9} / \mathrm{l}\right) .^{4}$ For each individual inflammatory marker and score we estimated the sensitivity, specificity, likelihood ratios, positive predictive value (PPV) and the area under the curve along with $95 \%$ confidence intervals using the DIAGT Stata module. We present PPVs for age-groups incorporated into clinical guidance.

\section{RESULTS}

Of 63,973 people with a code for unexpected WL, 12,024 (18.8\%) had all five inflammatory markers recorded; 5,214 (45.0\%) were men, median age 63 years (interquartile range, $47-76$ years) and 6612 (55.0\%) were women, 66 years (45-79 years). There were 217 (1.80\%) people diagnosed with cancer within 6 months, of whom $191(88.0 \%)$ were aged $\geq 60$ years.

The highest AUCs for individual inflammatory markers were for CRP (0.76 (95\% Cl 0.73-0.79)) and neutrophils (0.64 (0.61-0.67)) (Table 1). Scores with the greatest AUCs were $\mathrm{mGPS}=2(0.64$ $(0.61-0.67))$ and $\mathrm{NLS}=2(0.58(95 \% \mathrm{Cl} 0.55-0.60))$. The testing strategy with highest specificity was PLS $=2 \quad(98.65 \%$ (98.43-98.85\%) followed by NLS=2 (96.73\% (96.39-97.04\%)). Raised platelets and all scores had a positive likelihood ratio $\geq 5.0$, the rule of thumb to identify a good rule-in test. ${ }^{9}$ The testing strategy with highest sensitivity was a raised CRP $(70.97 \%$ (64.44-76.91\%)) followed by low lymphocytes $(46.54 \%(95 \% \mathrm{Cl}$ $39.76-53.42 \%)$. No normal maker or score had a negative likelihood ratio $<0.2$, the rule of thumb to identify a reliable rule-out test. $^{9}$

For most age-groups with unexpected WL, the PPV for a single abnormal inflammatory marker or a raised score was above $3 \%$. PPVs for individual abnormalities were lower than when they were combined as a score. For example, the PPV for cancer increased from $7.06 \%(95 \% \mathrm{Cl} 5.43-8.99 \%)$ for low albumin and $6.26 \%$ (5.34-7.29\%) for raised CRP to $8.85 \%(7.01-10.99 \%)$ when both were combined as $\mathrm{mGPS}=2$. While 2408 fewer people with unexpected WL would be referred unnecessarily per 100,000 people tested, using an $\mathrm{mGPS}=2$ compared to a raised CRP, 125 fewer people with cancer would be referred for investigation.

\section{DISCUSSION}

Summary

Our findings support the use of individual inflammatory marker abnormalities, in particular a raised CRP, in preference to inflammatory marker scores to triage people attending primary care with unexpected WL for cancer investigation.

Strengths and limitations

We ensured a consistent study population by selecting participants with all five inflammatory markers recorded. This strategy is likely to have resulted in an enriched population judged to be at higher risk of cancer. Further research is necessary to establish the generalisability of our findings. The outcome was all stages and types of cancer combined with follow-up limited to the period for which the association between unexpected $\mathrm{WL}$ and cancer diagnosis has been reported. ${ }^{8}$ A larger sample size would have permitted stratification by cancer site and stage to inform the investigative approach.

Previous literature

Without taking symptoms into account, an AUC of $0.64(95 \% \mathrm{Cl}$ $0.63-0.65)$, sensitivity of $46.1 \%(44.0-48.1)$ and specificity of $75.4 \%$

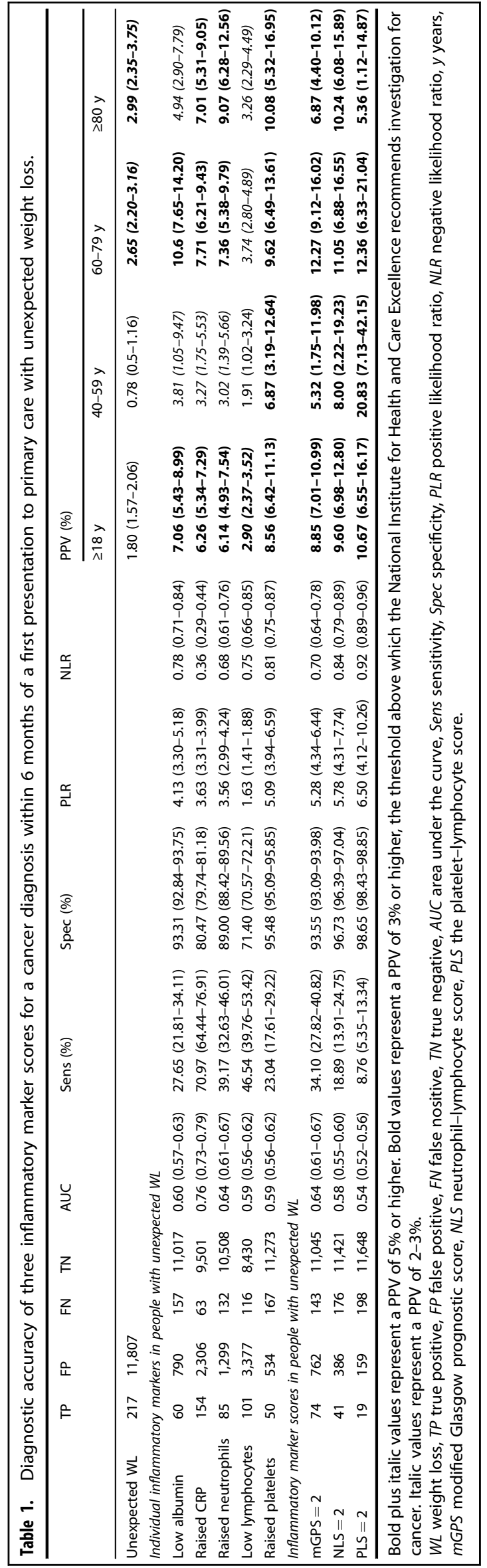


(74.1-75.6\%) was reported for a cancer diagnosis within 1 year of a $\mathrm{CRP} \geq 7 \mathrm{mg} / \mathrm{l}$ in a cohort of 111,440 people tested in primary care. ${ }^{10}$ Despite using a shorter follow-up period and higher CRP threshold, the AUC for CRP in our cohort was higher with increased sensitivity and specificity. Adding hypalbuminaemia to create an $\mathrm{mGPS}=2$ markedly lowered sensitivity and elevated specificity showing the rule-in potential of paired abnormalities.

\section{Implications}

Single inflammatory marker abnormalities are associated with an increased risk of cancer in people attending primary care with unexpected $\mathrm{WL}$, above the current threshold for investigation used in English guidelines, and especially in people aged $\geq 60$ years. If scores including pairs of abnormal inflammatory markers were instead used to select patients for cancer investigation, fewer people would be investigated to diagnose one cancer, with fewer false positives, but fewer people with cancer would be diagnosed overall. For example, there were 159 true positives for abnormal CRP alone, but 74 true positives if using $\mathrm{mGPS}=2$ combining raised CRP and low albumin. It is possible that combinations of multiple normal inflammatory markers hold greater potential to rule-out cancer than pairs of normal results. This approach warrants further investigation in clusters of symptomatic patients as it would mirror the reassurance taken from panels of normal tests by GPs and patients in clinical practice.

\section{CONCLUSION}

In people attending primary care with unexpected weight loss individual inflammatory marker abnormalities, notably a raised CRP, are more sensitive than inflammatory marker scores. The associated risk of cancer is high enough to justify cancer investigation.

\section{ACKNOWLEDGEMENTS}

We thank to Julie McClellan for critical review and advice during drafting. This study is based on data from the Clinical Practice Research Datalink obtained under licence from the UK Medicines and Healthcare Products Regulatory Agency. The data are provided by patients and collected by the NHS as part of their care and support. The interpretation and conclusions contained in this study are those of the authors alone.

\section{AUTHOR CONTRIBUTIONS}

B.D.N. conceived the study and has oversight as principal Investigator, designed the protocol, developed the code lists for use in the study, conducted the data management and the statistical analysis and wrote the first draft of the manuscript. J. L.O. advised on statistical methodology. B.D.N., J.L.O., P.A., W.T.H. and F.D.R.H. were involved in discussions throughout the design and analysis stages and in the drafting and commenting on further revisions of the manuscript. All authors read and approved the final manuscript.

\section{ADDITIONAL INFORMATION}

Ethical approval and consent to participate This study was approved by the Independent Scientific Application Committee for MHRA database research (ISAC) protocol number 16_164A2A. The study was performed in accordance with the Declaration of Helsinki. Consent to publish Not applicable.

Consent to publish Not applicable.

Data availability This study is based on CPRD data and is subject to a full licence agreement, which does not permit data sharing outside of the research team.
Competing interests The authors declare no competing interests.

Funding information B.D.N. is supported by National Institute for Health Research (NIHR) Doctoral Research Fellowship number (DRF-2015-08-18). P.A. is an NIHR senior investigator and is funded by NIHR Oxford Biomedical Research Centre and the NIHR Applied Research Collaboration (ARC) for Oxford and Thames Valley. W.H. is coPrincipal Investigator of the multi-institutional CanTest Research Collaborative funded by a Cancer Research UK Population Research Catalyst award (C8640) A23385). F.D.R.H. acknowledges his part-funding from the National Institute for Health Research (NIHR) School for Primary Care Research, the NIHR ARC for Oxford and Thames Valley, the NIHR Oxford Biomedical Research Centre (BRC, UHT) and the NIHR Oxford Medtech and InVitro Diagnostics Co-operative (MIC). The views expressed are those of the authors and not necessarily those of the NHS, the NIHR or the Department of Health.

Publisher's note Springer Nature remains neutral with regard to jurisdictional claims in published maps and institutional affiliations.

\section{REFERENCES}

1. Watson, J., Mounce, L., Bailey, S. E., Cooper, S. L. \& Hamilton, W. Blood markers for cancer. BMJ 367, 15774 (2019).

2. Koshiaris, C. et al. Early detection of multiple myeloma in primary care using blood tests: a case-control study in primary care. Br. J. Gen. Pract. 68, e586-e593 (2018).

3. Næser, E., Møller, H., Fredberg, U., Frystyk, J. \& Vedsted, P. Routine blood tests and probability of cancer in patients referred with non-specific serious symptoms: a cohort study. BMC Cancer 17, 817 (2017).

4. Dolan, R. D. et al. The prognostic value of systemic inflammation in patients undergoing surgery for colon cancer: comparison of composite ratios and cumulative scores. Br. J. Cancer 119, 40-51 (2018).

5. Baracos, V. E., Martin, L., Korc, M., Guttridge, D. C. \& Fearon, K. C. H. Cancerassociated cachexia. Nat. Rev. Dis. Prim. 4, 17105 (2018).

6. Nicholson, B. D. et al. Prioritising primary care patients with unexpected weight loss for cancer investigation: diagnostic accuracy study. BMJ 370, m2651 (2020).

7. Herrett, E. et al. Data resource profile: Clinical Practice Research Datalink (CPRD). Int. J. Epidemiol. 44, 827-836 (2015).

8. Nicholson, B. D. et al. The association between unexpected weight loss and cancer diagnosis in primary care: a matched cohort analysis of 65,000 presentations. Br. J. Cancer 122, 1848-1856 (2020).

9. Jaeschke, R., Guyatt, G. H. \& Sackett, D. L. Users' guides to the medical literature. III. How to use an article about a diagnostic test. B. What are the results and will they help me in caring for my patients? The Evidence-Based Medicine Working Group. JAMA 271, 703-707 (1994).

10. Watson, J., Salisbury, C., Banks, J., Whiting, P. \& Hamilton, W. Predictive value of inflammatory markers for cancer diagnosis in primary care: a prospective cohort study using electronic health records. Br. J. Cancer 120, 1045-1051 (2019).

Open Access This article is licensed under a Creative Commons Attribution 4.0 International License, which permits use, sharing, adaptation, distribution and reproduction in any medium or format, as long as you give appropriate credit to the original author(s) and the source, provide a link to the Creative Commons license, and indicate if changes were made. The images or other third party material in this article are included in the article's Creative Commons license, unless indicated otherwise in a credit line to the material. If material is not included in the article's Creative Commons license and your intended use is not permitted by statutory regulation or exceeds the permitted use, you will need to obtain permission directly from the copyright holder. To view a copy of this license, visit http://creativecommons. org/licenses/by/4.0/.

(c) The Author(s) 2021 